\title{
Overview on Determination of Elastic and Damping Properties of Different Materials using Impulse Excitation Technique
}

Inês Pereira

CEMMPRE, Department of Metallurgical and Materials Engineering, Faculty of Engineering, University of Porto, Porto, Portugal (up201105626@fe.up.pt)

\begin{abstract}
Knowledge of elastic and damping properties of materials is very relevant for the analysis and design of components, as they are relevant parameters in the performance of structural materials. The impulse excitation technique is a renowned dynamic technique for measuring dynamic elastic properties as Young's modulus, shear modulus and Poisson's ratio, as well as damping properties. This paper provides a review on the applicability of the impulse excitation technique in the analysis of elastic and damping properties of different types of materials.
\end{abstract}

Keywords: Impulse Excitation Technique, Resonant Frequency, Elastic Properties, Damping

Type: Review Article

๑ Open Access $[\mathbf{6}$ Peer Reviewed $@$ (i) CC BY

\section{Introduction}

A suitable determination of the elastic constants of a material allows the microstructural analyse of a solid, this is important because may set relevant inputs for structural calculus in engineering design. The measurement of this constants can be achieve by several experimental methods, and the most usually used are classified as static, like tensile and compressive mechanical stress tests and flexural loading tests, or dynamic techniques, as resonance techniques (Montecinos, Tognana, and Salgueiro 2016; Tognana et al. 2010).

One of the dynamic techniques used to measure resonant frequencies and consequently to determine the elastic properties of solids is the impulse excitation technique. The wide field of application of this technique can be divided on different domains of research: the internal crystal structure, properties and analysis of damage of the material and can verify complete systems (Brebels and Bollen 2015; Psiuk et al. 2016; Roebben et al. 1997).

The impulse excitation technique is based on the analysis of the vibration of a specimen. This is mechanically excited to make it vibrate at one or more of its resonant frequencies through a single-elastic strike with an impulse tool. A transducer, like a microphone with an adequate frequency range, detect the sound produced by the vibration of the specimen and then the signals are analysed by a sound processor software originating amplitude of the vibration as a function of time. Each one of them is analysed to obtain the resonant frequencies using the fast Fourier transform. The resonant frequencies of a solid are related to its mass, dimensions and elastic properties, this enables the determination of the values of elastic properties of the material from resonant frequencies by using analytical equations described in international standards, the elastic properties from. Moreover, the method allows determine the damping parameters of vibration, like loss factor and internal friction, the 
latter represents the energy absorption by the material and can be deduced from the amplitude decay of a free vibration. The internal friction, in contrast to resonant frequencies, is independent of the geometry of the solid and therefore can be used as additional information in process control (Brebels and Bollen 2015; Heritage, Frisby, and Wolfenden 1988; Lima 2014; Lopes et al. 2000; Psiuk et al. 2016; Tognana et al. 2010; Roebben et al. 2002; Roebben et al. 2000; Roebben et al. 1997).

The analysis can be conducted at room, cryogenic or high temperatures. In high temperatures tests other mechanisms are used to realise the excitation of the sample since this one can't be tapped directly in an oven (Schmidt, Alpern, and Tilgner 2005; Roebben et al. 1997).

As mentioned elastic properties, as Young's modulus, shear modulus and Poisson's ratio can be measured by using impulse excitation technique. Each elastic property is related with fundamental vibration modes, thus the flexural and longitudinal vibration modes are related to the Young's modulus in the longitudinal direction and the torsion mode to the shear modulus, for isotropic materials, the Poisson's ratio can be calculated from an analytical equation that relate Young's and shear modulus. The measured vibration modes depend on the geometry of the specimen, the location of the impulse and the transducer and the type of supports, the latter are situated in the nodes of the intended vibration mode to minimize frictional losses due to the supports and allow the specimen vibrate freely (Brebels and Bollen 2015; Roebben et al. 1997).

The method presented has advantages as: essentially non-destructive, tests procedure simplicity, more cost effective and quickness, minimal specimen preparation, small quantities of material needed, precise data, and tests can be done at high temperatures and aggressive atmospheres (Latella and Humphries 2004; Roebben et al. 1997; Montecinos, Tognana, and Salgueiro 2016; Schmidt, Alpern, and Tilgner 2005).

In the present work, a review on the applicability of the impulse excitation technique in the analysis of material properties, as elastic and damping properties, of different types of materials is presented, moreover the advantages and limitation in each paper reviewed are described, finishing the paper with a summary and conclusions about the potentialities of impulse excitation technique on characterization of different types of materials.

\section{Review of papers}

As presented by Voiconi et al. (2014) the elastic (Young's modulus and shear modulus) and damping properties of ductile aluminium foam with various densities was determined by using a non-destructive testing device to determine the resonant frequency of materials; the properties results for the vibration of the samples in flexural and torsional mode. The results show that Young's modulus and shear modulus increases with increasing density for both types of vibration modes. The results also show that the internal friction coefficient increase with decreasing relative density.

Heritage, Frisby, and Wolfenden (1988) developed a new method to obtain dynamic modulus as a function of temperature by the adaptation of the impulse excitation technique. The values of modulus were obtained by measuring the resonant frequency of flexural vibration at moderate temperatures of specimens tapped inside an electric furnace, with the microphone to analyse sound waves strategic placement outside the furnace. The results showed the imprecision in the value of the modulus was $\pm 1 \%$ in measurements made at different temperatures on a pure aluminium specimen when directly compared with data 
obtained on the same sample using another technique. As well, measurements at room temperature agreed with published data.

Tognana et al. (2010) study the dependence of the Young's modulus on the inclusion of three different volumetric filler content (aluminium, quartz or copper) in an matrix-epoxy composite. In this paper, was developed an experimental set-up using the concept of the impulse excitation technique where the spectrums obtained were analysed to determine the resonant frequencies and subsequently calculated the values of modulus. The results demonstrate that Young's modulus increase when the filler content increases, the authors attributed this behaviour to the higher Young's modulus of each material used as filler when compared to the Young's modulus of the epoxy matrix.

As reported by Montecinos, Tognana, and Salgueiro (2016) the Young's modulus of CuAlBe shape memory alloys was carry out by using the impulse excitation technique, where was verified the potentialities of this technique in alloys with a complex microstructure. The experimental device was developed and an evaluation of its performance was done by measurements of the Young's modulus in samples commonly used (commercial aluminium and copper), the values obtained were in agreement with those reported in the literature. Was also evaluated the CuAlBe samples using different vibration modes and the results show that the flexural and longitudinal modes attained the same value of the modulus. Was also found a strong dependence of Young's modulus with the grain size, where Young's modulus decreases for higher grain sizes.

As presented by Psiuk et al. (2016) the impulse excitation technique was used as a promising method for investigating the process of in situ ceramic slurry gelcasting, samples with different chemical compositions and different gelcasting characteristics were analysed. The impulse excitation technique was used since changes in slurry viscosity are related by alterations to the parameters of damping of the wave propagation in the medium (slurry). The results show that the measurements obtained with the equipment device based on impulse excitation technique are consistent with the ones reached by a viscometer and allowed formulating identical conclusions.

Marešová, Džugan, and Procházka (2012) determined the Young's modulus using three different methods, two of them allow the determination of static modulus (standard tensile tests and micro tensile tests with Digital Image Correlation evaluation) and the other the dynamic modulus, in this method the values were evaluated by analysis of free vibrations of a rectangular specimen after a tap (impulse excitation). The results show that was excellent agreement, for all methods, in elastic modulus values for longitudinal direction. While, for transversal direction the values of modulus were lower for both tensile tests, but not detected by dynamic evaluation. The authors concluded that the reason was the relatively larger size of the sample, when compared to other samples used in this work that lead to the need for averaging the values of properties.

As reported by Lopes et al. (2000) the impulse excitation of vibration method was used to evaluate dynamic Young's and shear modulus of glass-reinforced hydroxyapatite composites. The dependence of these two elastic properties on microstructural characteristics of the composites was assessed and establish an exponential dependence of these elastic properties with porosity. The results show that Young's modulus increased with sintering temperature, the authors concluded that the modulus increases because an increase in densification (porosity reduction) was observed with a rise of sintering temperature, since the increase of modulus depends on porosity reduction. 
Lima (2014) determined the damping capacity of the Cu-14Al-4Ni alloy, which was hot rolled and thermally annealed, by impulse excitation technique. The values obtained were compared with those of CuAlBe and NiTi alloys. The results show that Cu-14Al-4Ni alloy presented a damping level significantly higher than the CuAlBe and NiTi alloys, moreover the Cu-14Al-4Ni which was thermally annealed exhibited a higher damping capacity when compared with $\mathrm{Cu}-14 \mathrm{Al}-4 \mathrm{Ni}$ which was hot rolled.

Hauert, Rossoll, and Mortensen (2009) measured with impulse excitation technique the values of Young's modulus of particulate metal matrix composites with a high volume fraction of reinforcement. When compared, the experimental results were well described by analytical models that are used in the literature to describe the elastic behaviour of this type of material.

Roebben et al. (2000) performed tests using an equipment based on impulse excitation technique for high-temperatures on silicon nitride and zirconia ceramics, both materials present an internal friction behaviour with a great relevance to their structural performance. The results showed that the tests on silicon nitride allows quantify the effect of the glass transition and crystallisation of amorphous intergranular phases. Relatively to zirconia material the results showed that the effects of composition, even minimal differences in composition affected the measured internal friction, on the occurrence and extent of martensitic transformation can be investigated by the tests performed.

Latella and Humphries (2004) evaluated and compared the values of dynamic Young's modulus of a $2.25 \mathrm{Cr}$-1Mo steel and creep-damaged $2.25 \mathrm{Cr}$-1Mo steel at high temperature using the impulse excitation technique. Both steels presented a reduction of the Young's modulus with increasing temperature. This allowed the achievement of important information for structural design because the Young's modulus is a key parameter in toughness and thermal shock relations and in measurements of residual stresses, being crucial in monitoring the performance of high temperature structural steels. However, was detected that oxidation effects during the tests had a negative effect on the measurements, being a disadvantage of perform high temperature tests. The authors concluded that results obtained with the improved accuracy of the technique used in this work allowed a superior reliability and accuracy of materials data that can be use in structural design calculations and modelling.

As presented by Schmidt, Wicher, and Tilgner (2005) the impulse excitation technique was investigated to verify is applicability to measure the Young's modulus of moulding compounds, comparing the obtained values with data from other method, three point bending test. The results showed a high reproducibility when measurements on samples produced previously were performed, since a shift in the values detected the shifting of the manufacturing process. Moreover, the behavior of the modulus as function of temperature and moisture storage was investigate and was observed that the method is so precise that even small temperature differences during measuring allowed effects on the values of the modulus, was showed that values of Young's modulus reduced with moisture and increased with temperature.

As presented by Dal Bó et al. (2017) was studied the Young's modulus of porcelain using the impulse excitation technique at room temperature up to higher temperatures with the aim of knowing the influence of quartz particle size, weight content and firing temperature on the measured modulus. The porcelain microstructure was simulated by developing an albite glass matrix with added crystalline quartz particles. The results showed that quartz particles have a relevant influence on the elastic modulus and correlations were established, with 
those can be achieved a prediction of the mean particle size and a semi-quantitative estimation of the volume fraction of quartz by using Young's modulus data.

\section{Conclusion}

In this paper was showed the potentialities of application of impulse excitation technique in domains research as material properties and monitoring manufacturing process. Moreover, it was observed in the several papers that the method can be used to analyse different type of materials, as metals, ceramics and composites.

It was discussed that the impulse excitation technique: analyse materials with complex microstructure; allows the characterization of the elastic behaviour as a function of temperature since the tests can be done at room and high temperatures. In cases of high work temperature materials this can be particularly useful because it's possible to analyse the performance of the material at high temperatures. Characterization of phase transformations was also reported. Further, this simple, low cost and non-destructive technique; has a higher reproducibility, when compared with other methods to determine Young's modulus, allowing monitoring drifts of the manufacturing process and detecting effects on Young's modulus even with small differences of temperature. It was also seen that Young's modulus data can be used for prediction of volume fraction and size of particles.

The impulse excitation technique is an important method to describe a material's behaviour and can be applied in several research domains and on different types of materials, allowing the use of this knowledge in analysis, research and engineering design of components.

\section{References}

Brebels, Adriaan and Bart Bollen. 2015. "Non-destructive evaluation of material properties as function of temperature by the Impulse Excitation Technique". The e-Journal of Nondestructive Testing 20 (6). Accessed March 27, 2017. http://www.ndt.net/article/ndeindia2014/papers/CP0023_full.pdf.

Dal Bó, Marcelo, Vicente Cantavella, Enrique Sanchéz, Francisco A. Gilabert, Anselmo O. Boschi and Dachamir Hotza. 2017. "An estimate of quartz content and particle size in porcelain tiles from young's modulus measurements". Ceramics International 43 (2):22332238. Accessed April 7, 2017. DOI: 10.1016/j.ceramint.2016.10.204.

Hauert, Aude, Andreas Rossoll and Andreas Mortensen. 2009. "Young's modulus of ceramic particle reinforced aluminium: Measurement by the Impulse Excitation Technique and confrontation with analytical models". Composites Part A: Applied Science and Manufacturing 40 (4):524-529. Accessed April 6, 2017. DOI: 10.1016/j.compositesa.2009.02.001.

Heritage, Kevin, Clayton Frisby and Alan Wolfenden. 1988. "Impulse excitation technique for dynamic flexural measurements at moderate temperature". Review of Scientific Instruments 59 (6):973-974. Accessed March 26, 2017. DOI: 10.1063/1.1139761.

Latella, Bruno A. and Samuel R. Humphries. 2004. "Young's modulus of a $2.25 \mathrm{Cr}-1 \mathrm{Mo}$ steel at elevated temperature". Scripta Materialia 51 (7):635-639. Accessed April 6, 2017. DOI: 10.1016/j.scriptamat.2004.06.028.

Lima, Pedro Cunha de. 2014. "Obtenção e determinação da capacidade de amortecimento da liga Cu-14Al-4Ni pelo método de excitação por impulso". Dissertação de Mestrado, Faculdade do Gama, Universidade de Brasília. Accessed March 28, 2017. http://repositorio.unb.br/handle/10482/15686. 
Lopes, Maria A., Rui F. Silva, Fernando J. Monteiro and José D. Santos. 2000. "Microstructural dependence of Young's and shear moduli of P 205 glass reinforced hydroxyapatite for biomedical applications". Biomaterials 21 (7):749-754. Accessed March 22, 2017. DOI: 10.1016/S0142-9612(99)00248-3.

Marešová, Martina, Jan Džugan and Radek Procházka. 2012. "Youngs modulus determination for spring back evaluation of cold formed parts". 2nd International Conference on Recent Trends in Structural Materials. Accessed April 4, 2017. http://comat2012.tanger.cz/files/proceedings/11/reports/1247.pdf.

Montecinos, S., S. Tognana and W. Salgueiro. 2016. "Determination of the Young's modulus in CuAlBe shape memory alloys with different microstructures by impulse excitation technique". Materials Science and Engineering: A 676:121-127. Accessed April 2, 2017. DOI: 10.1016/j.msea.2016.08.100.

Psiuk, Bronisław, Paulina Wiecinska, Barbara Lipowska, Emilia Pietrzak and Jacek Podwórny. 2016. "Impulse excitation technique IET as a non-destructive method for determining changes during the gelcasting process". Ceramics International 42 (3):3989-3996. Accessed March 31, 2017. DOI: 10.1016/j.ceramint.2015.11.067.

Roebben, Gert, Bikramjit Basu, Jef Vleugels, Jan Van Humbeeck and Omer Van der Biest. 2000. "The innovative impulse excitation technique for high-temperature mechanical spectroscopy". Journal of Alloys and Compounds 310 (1):284-287. Accessed April 6, 2017. DOI: 10.1016/S0925-8388(00)00966-X.

Roebben, Gert, B Bollen, A Brebels, Jan Van Humbeeck and Omer Van der Biest. 1997. "Impulse excitation apparatus to measure resonant frequencies, elastic moduli, and internal friction at room and high temperature". Review of Scientific Instruments 68 (12):4511-4515. Accessed April 6, 2017. DOI: 10.1063/1.1148422.

Roebben, Gert, Ren-Guan Duan, Diletta Sciti and Omer Van der Biest. 2002. "Assessment of the high temperature elastic and damping properties of silicon nitrides and carbides with the Impulse Excitation Technique". Journal of the European Ceramic Society 22 (14):25012509. Accessed April 6, 2017. DOI: 10.1016/S0955-2219(02)00111-5.

Schmidt, Roland, Peter Alpern and Rainer Tilgner. 2005. "Measurement of the Young's modulus of moulding compounds at elevated temperatures with a resonance method". Polymer Testing 24 (2):137-143. Accessed April 6, 2017. DOI: 10.1016/j.polymertesting.2004.09.009.

Schmidt, Roland, Viktor Wicher and Rainer Tilgner. 2005. "Young's modulus of moulding compounds measured with a resonance method". Polymer Testing 24 (2):197-203. Accessed April 6, 2017. DOI: 10.1016/j.polymertesting.2004.08.010.

Tognana, Sebastián, Walter Salgueiro, Alberto Somoza and Angel Marzocca. 2010. "Measurement of the Young's modulus in particulate epoxy composites using the Impulse Excitation Technique". Materials Science and Engineering: A 527 (18):4619-4623. Accessed April 2, 2017. DOI: 10.1016/j.msea.2010.04.083.

Voiconi, T., L. Marsavina, E. Linul and J. Kováčik. 2014. "Determination of elastic and damping properties for closed-cell aluminium foams using Impulse Excitation Technique". Paper presented at XIIIth Youth Symposium of Experimental Solid Mechanics, in Czech Republic. June 29th - July 2nd. Accessed April 3, 2017. https://www.researchgate.net/profile/Emanoil_Linul/publication/292398927_Determina tion_of_Elastic_and_Damping_Properties_for_Closed- 
Cell_Aluminium_Foams_Using_Impulse_Excitation_Technique/links/56e9439108aef53a1 2bd82e0.pdf.

\section{Acknowledgements}

The author acknowledges the support of SAKTHI, Portugal, S.A., IK4-Azterlan and Center for Innovation and Technology N. MAHALINGAM, under the scope of Brake Noise project. 